\title{
Algoritmo Genético aplicado ao problema de alocação/localização de facilidades.
}

\author{
Tarcísio Barroso Marques ${ }^{1}$, Otho Garcia da Silva Neto ${ }^{1}$, Daniel da Silva Diniz ${ }^{1}$ \\ ${ }^{1}$ Instituto Federal de Educação, Ciência e Tecnologia Fluminense (IF Fluminense) \\ Campus Itaperuna - 28.300-000 - Itaperuna - RJ - Brasil \\ tarcisio.marques@iff.edu.br, othogar@gmail.com, danielsdnz@gmail.com
}

\begin{abstract}
This article presents the Genetic Algorithm (GA) applied to the problem of allocation / location of health center. The goal is a minimum number of health posts covering a greater quantity of demand points by positioning them geographically at strategic points. In this problem, the number of facilities is not fixed, and it is up to GA to find a number of health centers that meet the established criteria. The possibility of filament generation during times of movement at health posts is disregarded. The Genetic Algorithm proved to be very effective in computational tests in several small instances of large problems, with a rapid evolution of the population.
\end{abstract}

Resumo. Este artigo apresenta o Algoritmo Genético (AG) aplicado ao problema de alocação/localização de postos de saúde. O objetivo é alocar um número mínimo de postos de saúde cobrindo a maior quantidade de pontos de demanda posicionando-os geograficamente em pontos estratégicos. Neste problema, o número de facilidades não é fixado, cabendo ao AG encontrar um número de postos de saúde que atenda aos critérios estabelecidos. São desconsideradas a possível geração de filas em dias de mais movimento nos postos de saúde. O Algoritmo Genético mostrou-se muito eficaz em testes computacionais realizados nas diversas instâncias de problemas de pequeno à grande porte, com uma rápida evolução da população.

\section{Introdução}

Decisões sobre aonde alocar ou instalar facilidades considerando as necessidades do usuário, são entendidas como problemas de localização de facilidades, onde, "facilidades" se entende por postos de saúde, depósitos, escolas, fábricas etc., enquanto clientes refere-se a bairros, unidades de vendas, pacientes etc. O processo decisório de alocação é algo complexo de ser analisado, principalmente quando envolve um grande número de possibilidades, onde às vezes se faz necessário efetuar cálculos enormes que resultam em números exorbitantes, sendo necessário anos para efetuar tais cálculos. "A maioria dos Problemas de Localização de Facilidades é considerada de difícil solução, pertencendo alguns deles à classe NP-difícil.” (LOUREIRO, 2012, 1301).

Os problemas de localização são de natureza combinatória, pois consistem em selecionar de um conjunto discreto e finito de dados o melhor subconjunto que satisfaça determinados critérios."(Arroyo, 2006, 1346) Este problema é chamado de p-medianas. "O problema das p-medianas consiste em determinar a localização, em uma rede, de $p$ 
facilidades (medianas) de um conjunto pré-definido $n(n>p)$, minimizando-se a soma de todas as distâncias de cada ponto de demanda à sua mediana mais próxima."(Rosário, 2002, 3).

A pesquisa apresentada neste artigo fomentada pelo Instituto Federal Fluminense Campus Itaperuna-RJ, apresenta a proposta de uma heurística baseada em algoritmo genético, que visa definir qual a quantidade e o melhor posicionamento das facilidades. Como parâmetros iniciais são definidas apenas a quantidade máxima de facilidades que poderão ser utilizadas com o peso necessário, sendo que, através do peso o algoritmo irá definir a quantidade de facilidades que melhor o soluciona posicionando-os da melhor forma possível.

\section{Definição do Problema}

Nesta seção apresenta-se a definição do problema de localização de facilidades, abordado neste trabalho.

\section{Notações:}

$P=\{1, \ldots, n\}$ : conjunto de pontos de demanda (um ponto de demanda pode ser um bairro ou um quarteirão de um bairro por exemplo);

$C=\{1, \ldots, m\}$ : conjunto de pontos potenciais onde podem ser alocadas as facilidades (se no ponto $j \in C$ é alocada uma facilidade, então é dito que a facilidade $j$ é aberta, caso contrário a facilidade $j$ está fechada);

$\mathrm{A}=\{\mathrm{a} 1, \ldots, \mathrm{ak} \mid \mathrm{m} \geq \mathrm{k} \in \mathrm{C}\}$ : conjunto de facilidades abertas.

$\mathrm{B}=\{\mathrm{b} 1, \ldots, \mathrm{by} \mid \mathrm{m} \geq \mathrm{y} \in \mathrm{C}\}$ : conjunto de facilidades fechadas.

cj: variável de decisão $\in\{0,1\}$. Se a facilidade $j$ é aberta tem-se aj $=1$, caso contrário aj $=0$.

dij: distância (euclidiana) do ponto i $\in P$ com coordenadas(xi,yi)ao ponto j $\in A$ com coordenadas(xj,yj);

S: constante de normalização que irá privilegiar mais ou menos o uso das facilidades abertas, de acordo com o seu valor estipulado, devendo ser ajustada para cada tipo de problema.

Formulação:

$$
\text { Minimizar } f(x)=\sum_{i=1}^{n} \min \left\{d_{i j} \mid j \in A\right\}+S \cdot k \mid m \geq k \in C
$$

A função objetivo em $\mathrm{f}: \Omega \in \mathrm{R} \quad(\Omega=$ conjunto das soluções viáveis, $\mathrm{R}=$ conjunto dos números reais) busca aproximar os pontos de demanda às facilidades a serem abertas atendendo um ponto de demanda pi $\in \mathrm{P}$ pela facilidade aj $\in \mathrm{A}$ que esteja mais próxima. A função objetivo também será melhor à medida que menos facilidades são usadas, indicando uma economia dos gastos com a abertura dos postos de saúde.

\section{O Algoritmo Genético aplicado ao problema de alocação de facilidades.}

O Algoritmo Genético é uma das áreas da Inteligência artificial, sendo que seus métodos foram inspirados na teoria da evolução definida por Charles Darwin. "Os melhores indivíduos de um algoritmo genético tendem a sobreviver por mais tempo, e assim transportam o seu material genético às novas soluções que serão geradas no futuro.”(Barsanulfo, Allan, et al. 2016, 27). Com isso há uma seleção de dois indivíduos 
da população, causando maior probabilidade dos melhores indivíduos serem escolhidos, a próxima etapa é efetuar o cruzamento combinando uma parte do conteúdo de cada pai, gerando assim um filho, que em sua composição possui o conteúdo dos pais mesclados. Uma mutação é realizada onde se altera uma pequena porcentagem do conteúdo desse filho.

"A seleção, o cruzamento e a mutação são operadores genéticos que transformam a população através de sucessivas gerações. Após a aplicação desses operadores, ao longo das gerações, espera-se que os indivíduos da população convirjam para uma boa solução, não necessariamente a ótima."(SILVA, Anderson Freitas, 2006, 2).

\section{Testes Computacionais.}

Os testes computacionais consistiram na elaboração de instâncias de problemas de pequeno à grande porte, sendo comparados o valor final da função objetivo apresentada pelo Algoritmo Genético, com o valor final da função objetivo para o mesmo problema, abordado pela Busca Local com a permuta All Pairs, que consiste na busca de melhores soluções através da troca de pares, ou conjuntos de valores que trocam suas posições, gerando uma nova solução. A Tabela 1 exibe os testes realizados para $n$ pontos de demanda e $m$ locais candidatos à instalação dos $k$ postos de saúde, locados pelo problema. Em todas as instâncias foi adotada uma população contendo 500 indivíduos.

Tabela 1. Instâncias de problemas aleatórios

\begin{tabular}{|c|c|c|c|c|c|c|c|}
\hline № & $\begin{array}{c}\text { Problema } \\
n \_m \_k\end{array}$ & $\begin{array}{l}f(x) \text { Algor. } \\
\text { Genético }\end{array}$ & $\begin{array}{c}f(x) \\
\text { Busca } \\
\text { Local }\end{array}$ & № & $\begin{array}{c}\text { Problema } \\
n \_m \_k\end{array}$ & $\begin{array}{l}f(x) \text { Algor. } \\
\text { Genético }\end{array}$ & $\begin{array}{c}f(x) \text { Busca } \\
\text { Local }\end{array}$ \\
\hline 1 & 10_10_1 & 3157 & 13157 & 5 & 300_300_5 & 95481 & 97858 \\
\hline 2 & 20_20_1 & טעלוכו & 16850 & 6 & 400_400_6 & 104875 & 108954 \\
\hline 3 & 40_40_1 & 20999 & 20999 & 7 & 700_700_6 & 158698 & 172462 \\
\hline 4 & 200_200_3 & 64162 & 67458 & 8 & 1000_1000_7 & 208463 & 210240 \\
\hline
\end{tabular}

Comparando-se com a Busca Local, pode-se observar na Tabela 1 que o Algoritmo Genético obteve um melhor desempenho em $75 \%$ das instâncias de problemas analisadas, comprovando a sua eficiência.

A solução gráfica da instância de problema 6 está representada pela Figura 1 onde são plotados à esquerda o resultado obtido pelo Algoritmo Genético e à direita a solução encontrada pela Busca Local.
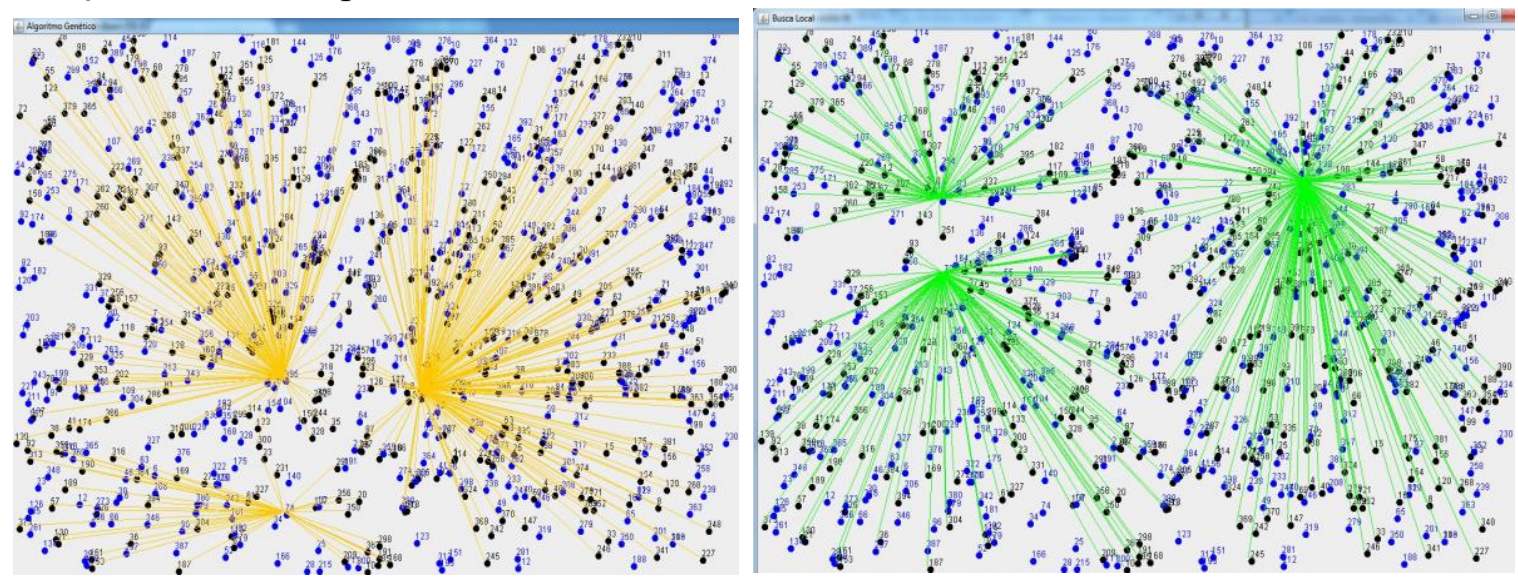


\section{Figura 1. Representação gráfica da instância de problema 6.}

Pode-se observar na Figura 1 que ambas as soluções utilizaram três facilidades. O Algoritmo Genético obteve um desempenho melhor porque aproximou mais as facilidades aos pontos de demanda, obtendo de uma forma geral, um menor somatório das distâncias envolvidas.

\section{Conclusão}

Neste trabalho procurou-se locar um número desconhecido de facilidades posicionandoas (alocando) de tal forma a minimizar ao máximo as distâncias envolvidas entre as facilidades abertas e os pontos de demanda atendidos.

Esta solução objetiva auxiliar a tomada de decisões, quando por exemplo é conhecido o custo da abertura de uma facilidade (custo para abrir e manter um posto de saúde por exemplo) mas não é sabido quantos postos devem ser abertos e onde eles devem ser posicionados.

De acordo com as instâncias de problemas gerados, o Algoritmo Genético apresentou melhores resultados em praticamente todos os problemas. Devido a complexidade do modelo tratado, uma vez que a população do Algoritmo Genético apresentou indivíduos contendo um número diferente de genes, observou-se um tempo computacional requerido pelo Algoritmo Genético em média 65\% superior ao tempo gasto pela busca local, considerando as mesmas instâncias de problemas e critérios de parada.

\section{Referências}

Silva, Anderson Freitas, and AC De Oliveira. "Algoritmos genéticos: alguns experimentos com os operadores de cruzamento ("Crossover") para o problema do caixeiro viajante assimétrico." Anais do XXVI ENEGEP-Encontro Nacional de Engenharia de Produção, Fortaleza (2006).

Barsanulfo, Allan, et al. "Escalonamento De Horários Acadêmicos Utilizando Algoritmos Genéticos." Jornal De Engenharia, Tecnologia E Meio AmbienteJetma 1.1 (2016): 27-31.

Arroyo, José Elias Cláudio, and Tarcísio Barroso Marques. "Heurística Grasp Aplicado ao Problema de Alocação de Antenas de Transmissão." XXXVIII Simpósio Brasileiro de Pesquisa Operacional, Goiânia-GO (2006).

Loureiro, Sérgio Adriano, Christiane Lima Barbosa, and Orlando Fontes Lima Jr. "Procedimento para localização e alocação de vagas de carga e descarga em centros urbanos." Anais do XXVI ANPET (2012).

do Rosário, Raimundo Ronilson Leal, Celso Carnieri, and Maria Teresinha Arns Steiner. "Proposta de solução para o problema das p-medianas na localização de unidades de saúde 24 horas." XXII Encontro Nacional de Engenharia de Produção, Curitiba (2002). 